\title{
Role of the ApxIB/ApxID exporter in secretion of the ApxII and ApxIII toxins in Actinobacillus pleuropneumoniae
}

\author{
Hye-Jin Yoo, Seungwoo Lee, Doug-Young Ryu* \\ Research Institute for Veterinary Science, College of Veterinary Medicine, Seoul National University, Seoul 08826, Korea
}

Abstract: Apx toxins are a virulent factor of Actinobacillus pleuropneumoniae (App). At least four genes, apxC, apxA, apxB, and $a p x D$, are involved in the release of Apx toxins from App. apxA encodes Apx toxins, whereas $a p x B$ and $a p x D$ encode exporters. Some serotypes of App such as serotype 2 retain apxIBD, apxIICA, and apxIIICABD. Although the specificity of the ApxIB/ApxID exporter to ApxII has been established in those serotypes, that to ApxIII is under-studied. We constructed an apxIB-and apxID-lacking mutant strain of the App serotype 2 to study whether the ApxIB/ApxID exporter is capable of secreting both ApxII and ApxIII toxins.

Keywords: Actinobacillus pleuropneumoniae, toxin, export, recombination

*Corresponding author

Doug-Young Ryu

Research Institute for Veterinary Science,

College of Veterinary Medicine, Seoul

National University, 1 Gwanak-ro, Gwanak-gu,

Seoul 08826, Korea

Tel: +82-2-880-1253

E-mail: dyryu@snu.ac.kr

ORCID

Hye-Jin Yoo

https://orcid.org/0000-0001-7239-0640

Seungwoo Lee

https://orcid.org/0000-0003-0634-2522

Doug-Young Ryu

https://orcid.org/0000-0002-4392-1227

Conflict of Interest

The authors declare no conflict of interest.

Received: May 26, 2020

Revise d: September 14, 2020

Accepted: September 17, 2020
Porcine pleuropneumonia is a contagious disease caused by Actinobacillus pleuropneumoniae (App) [1-3]. App is a Gram-negative bacterium that can be differentiated into 18 serotypes [4]. Several virulence factors are involved in the pathogenesis of App, including capsule polysaccharide, lipopolysaccharide, proteases, transferrin-binding proteins, outer membrane proteins, and Apx toxins [5]. All serotypes of App can variously produce four different Apx toxins, namely ApxI, ApxII, ApxIII, and ApxIV, which have potential value for use in vaccines and diagnostic tests. At least four genes, apxC, apxA, $a p x B$, and $a p x D$, are required for activated Apx toxins to be released from the bacterial cells $[1,2]$. The $a p x C$ gene encodes a protein that activates the preApx toxins by post-translational acylation. The apxA gene encodes the toxin structure. The $a p x B$ and $a p x D$ genes encode exporters that are capable of secreting Apx toxins [1,2].

Strains of App serotype 2 retain the apxIBD, apxIICA, and apxIIICABD genes [1,6]. Many ApxII-producing App serotypes such as serotype 2 can release this toxin via the ApxIB/ApxID exporter [1,7]. With the exception of serotype 3, all serotypes that do not produce ApxI possess a truncated apxI operon consisting of $a p x I B$ and $a p x I D$ and are capable of producing the ApxIB/ApxID exporter that functions in ApxII secretion. However, the function of the ApxIB/ApxID exporter in the secretion of ApxIII is relatively under-studied in those serotypes. In this study, we constructed a mutant strain of App serotype 2 (1536; American Type Culture Collection, USA) by inactivation of apxIB and apxID to analyze the specificities of the ApxIB/ApxID exporter to the ApxIIA and ApxIIIA toxins.

A pBKS-App-Kan ${ }^{\mathrm{r}}$ recombination vector was designed to contain a fragment of consecutively arranged $a p x I B$ and apxID genes (GenBank accession number X68595.1) that were inactivated by the introduction of $\mathrm{Kan}^{\mathrm{r}}$ gene (Fig. 1A). For the plasmid preparation, a $4.0 \mathrm{~kb}$-long fragment of the apxIB and apxID genes was amplified from 1536 genomic DNA by polymerase chain reaction (PCR) using the primers apxIBD-F and apxIBD-R (Table 1). The PCR product was cloned into pTOP TA V2 (Enzynomics, Korea) to produce pTOP-App. pTOP-App was digested with SpeI and Xhol, and the $4.1 \mathrm{~kb}$ insert was ligated into pBluescript II $\mathrm{KS}(+)$ (Stratagene, USA) to obtain pBKS-App. Escherichia coli DH5 $\alpha$ was used for plasmid DNA amplification. pBKS-App was digested with BamHI and MfeI to delete a 748 bp fragment of the apxIB and apxID genes. A fragment encoding $\mathrm{Kan}^{\mathrm{r}}$ was amplified 
Table 1. Sequences of oligonucleotide primers used for polymerase chain reaction

\begin{tabular}{llc}
\hline \hline Primer & \multicolumn{1}{c}{ Sequence (5’ to 3') } & Restriction site \\
\hline apxIB-A & GTA GGC TCA GGC GGA CCG TTA G & \\
apxIBD-F & TCT TTA GCA CAA GCA GCT TAA GAT AG & \\
apxIBD-R & AGC CGA TGG TTA GCT TTA AAT ACA GTA & BamHI underlined \\
apxID-A & CAT TAA ATT ACT TAG CAC GGC GGT AGC ATC AT \\
apxID-B & CGC CTT AAA CAA TTT AAT GAC AGT ATC GGG & MfeI underlined \\
KanR-F & AAG GAT CCG AGG ATG GTT TCG CAT GAT TGA & \\
KanR-R & CGC AAT TGA ACC TTT CAT AGA AGG CGG CGG \\
SP1 & GAT TCA GCA AGG TGA AGT GAT & \\
SP2 & GAT AAC GTT CCA ATT TAG TCA G & \\
\hline
\end{tabular}

A
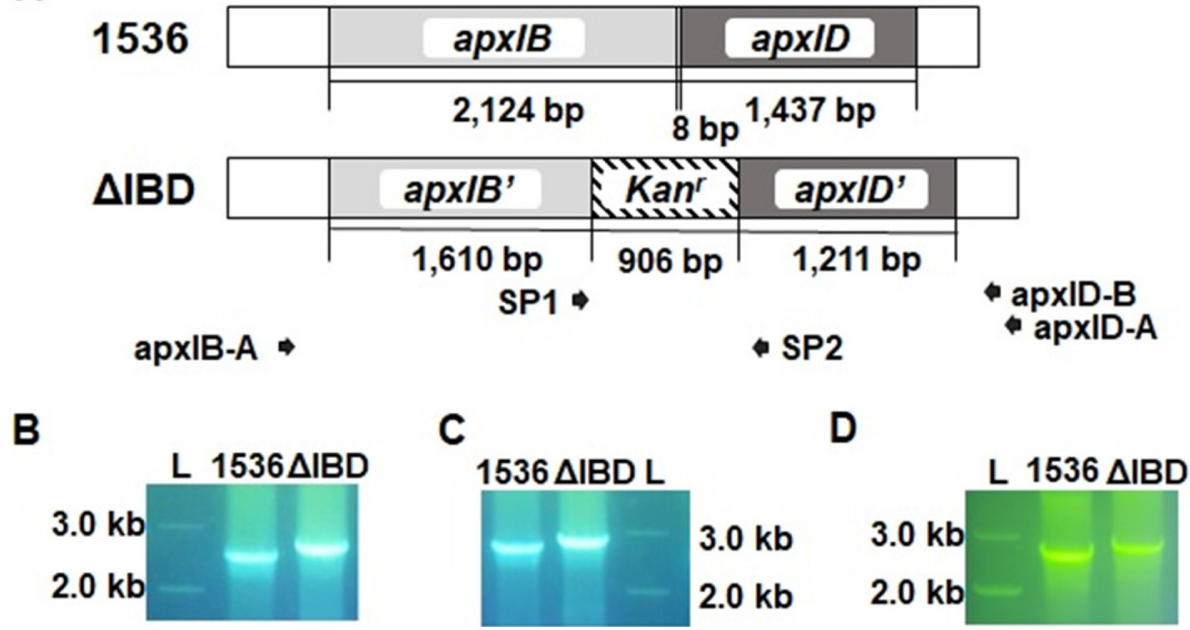

Fig. 1. Construction of a mutant $\triangle I B D$ strain. (A) Schematic structure of the apxIBD gene of the wild-type 1536 and mutant $\triangle I B D$ strains of App. (B to D) PCR analysis of genomic DNA from 1536 and $\Delta$ IBD using primer pairs SP1/apxID-B (B), SP1/apxID-A (C), and apxIB-A/SP2 (D). L, DNA ladder.

from pECFP-C1 (BD Biosciences, USA) using the primers KanR-F and KanR-R. The PCR product was inserted into the BamHI and MfeI digested pBKS-App to form pBKS-App-Kan ${ }^{r}$.

Transformation of the 1536 strain was performed with pBKS-App-Kan ${ }^{r}$ plasmid DNA linearized by SpeI. The App strains were cultured with PPLO-based media as described previously [8]. A transformant ( $\triangle \mathrm{IBD})$ was selected by PCR screening with three primer pairs, SP1/apxID-B, SP1/apxIDA, and apxIB-A/SP2 (Fig. 1B, 1C, and 1D). In order to confirm the recombination, the $\mathrm{PCR}$ amplicons for primer pairs SP1/apxID-B and apxIB-A/SP2 were sequenced with primers SP2 and SP1, respectively (data not shown).

To obtain the growth curves of $\triangle \mathrm{IBD}$, overnight cultures were diluted at 1:1,000 and grown for $12 \mathrm{~h}$. The $\mathrm{OD}_{600}$ of the culture was determined at intervals of $2 \mathrm{~h}$ with the Ultrospec 2000 UV/visible spectrophotometer (Pharmacia, Stockholm, Sweden). An obvious difference in the growth rate was observed between the $\triangle \mathrm{IBD}$ and 1536 strains (Fig. 2A). $\triangle \mathrm{IBD}$ showed a delayed growth rate compared to 1536, suggesting that the inactivation of the apxIB and apxID genes has a negative influence on the viability of App. The decreased growth rate might be due to cellular stress induced by unreleased Apx toxins. Despite the reduced growth rate, $\triangle \mathrm{IBD}$ was found to be genetically stable during 12 successive passages in culture (Fig. 2B).

To express a fusion recombinant protein named Apx132, a $1,345 \mathrm{bp}$ gene fragment was synthesized containing parts of ApxI (amino acids 460-578; GenBank accession number WP_005598583.1), ApxIII (amino acids 643-798; AAK 50053.1), and ApxII (amino acids 834-956; AAU84700.1). Fifteen-residue linkers coding for (GGGGS)3 were added between the ApxI and ApxIII parts and between the ApxIII and ApxII parts. The gene fragment was inserted into the linearized pET28a(+) (Novagen, UK), and the recombinant plasmid was then transformed into $E$. coli BL21 (DE3). Selected colonies were cultured at $37^{\circ} \mathrm{C}$ in LB media supplemented with $100 \mu \mathrm{g} / \mathrm{mL}$ kanamycin. Overnight cultures (diluted 1:100) were grown until mid-log phase at which point protein expression was induced for $4 \mathrm{~h}$ by the addition of isopropyl- $\beta$-D-thiogalactopyranoside to a final concentration of $1 \mathrm{mM}$. The cells were washed twice with ice-cold PBS (pH 7.4) and then lysed for $30 \mathrm{~min}$ in Buffer A (100 
A

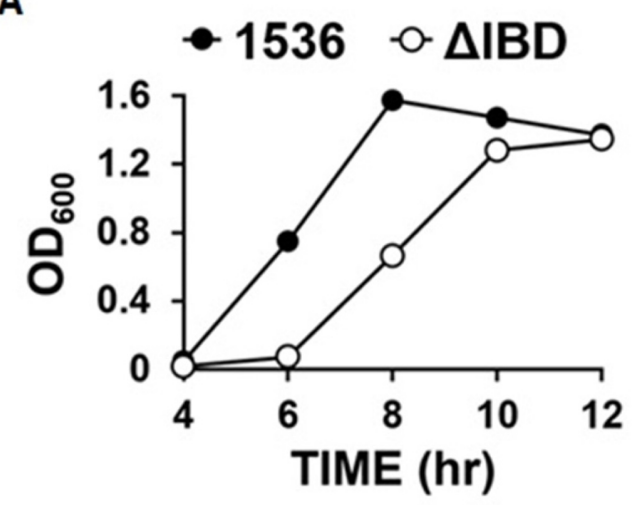

B

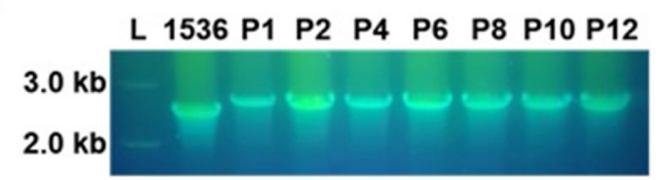

Fig. 2. Growth rate and stability. (A) $\triangle I B D$ exhibited a lower growth rate than 1536 in PPLO-based media. (B) $\triangle \mathrm{IBD}$ was genetically stable in 12 successive passages in the media. Genomic DNA was separated from 1536 and passages 1, 2, 4, 6, 8, 10, and 12 (P1, P2, P4, P6, P8, P10, and P12) of $\Delta \mathrm{IBD}$, and analyzed with PCR using a primer pair SP1/apxID-B. L, DNA ladder.

$\mathrm{mM}$ sodium phosphate, $10 \mathrm{mM}$ Tris- $\mathrm{HCl}, 100 \mu \mathrm{g} / \mathrm{mL}$ lysozyme and $5 \mathrm{mM}$ phenylmethanesulfonylfluoride (PMSF), $\mathrm{pH}$ 8.0). The lysate was centrifuged at $10,000 \times \mathrm{g}$ for $20 \mathrm{~min}$, and the resulting pellet was resuspended by incubation for $1 \mathrm{~h}$ at room temperature in Buffer B $(8 \mathrm{M}$ urea, $10 \mathrm{mM}$ Tris- $\mathrm{HCl}$, $100 \mathrm{mM}$ sodium phosphate and $5 \mathrm{mM}$ PMSF; $\mathrm{pH}$ 8.0). The solubilized extracts were then loaded onto a Ni-nitrilotriacetic acid column (Qiagen, USA). The column was washed with Buffer C (8 M urea, $10 \mathrm{mM}$ Tris- $\mathrm{HCl}, 100 \mathrm{mM}$ sodium phosphate and $5 \mathrm{mM}$ PMSF; pH 6.3) and eluted with Buffers $\mathrm{D}$ and $\mathrm{E}$ ( $8 \mathrm{M}$ urea, $10 \mathrm{mM}$ Tris- $\mathrm{HCl}, 100 \mathrm{mM}$ sodium phosphate and $5 \mathrm{mM}$ PMSF; pH 5.9 and 4.5, respectively).

Apx132 protein was purified with a Ni-nitrilotriacetic acid column (Qiagen) and used for the preparation of an antiApx132 antiserum in mice. Female Balb/c mice aged 6 to 8 weeks (SLC Japan, Japan) were housed in laboratory animal facilities at the College of Veterinary Medicine, Seoul National University. The mice were immunized by subcutaneous administration of $25 \mu \mathrm{g}$ of immunogen on Days 0,14 , 28 , and 42 . Immunogens were administered in complete Freund's adjuvant on Day 0 and in incomplete Freund's adjuvant on Days 14, 28, and 42. Serum samples were obtained by retro-orbital bleeding under anesthesia on Day 63. Western blots were performed with anti-Apx132 antiserum and horseradish peroxidase-conjugated goat anti-mouse immunoglobulin G secondary antibodies.

Precipitation of proteins secreted by the App strains into
A
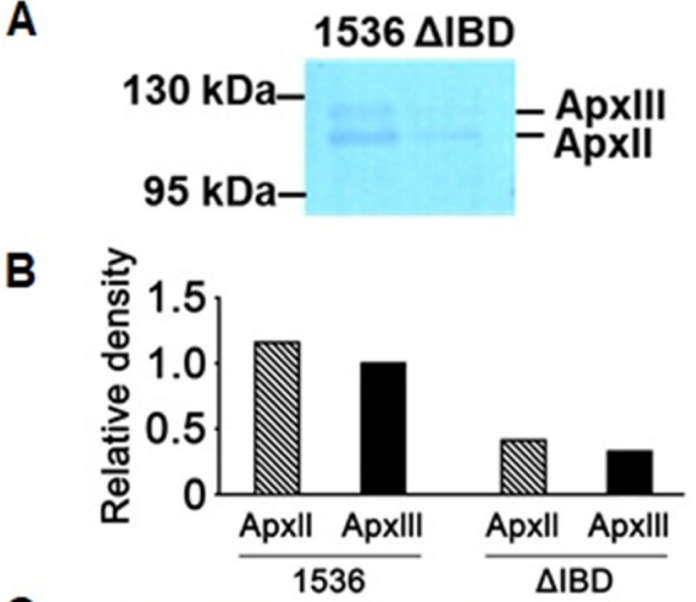

C

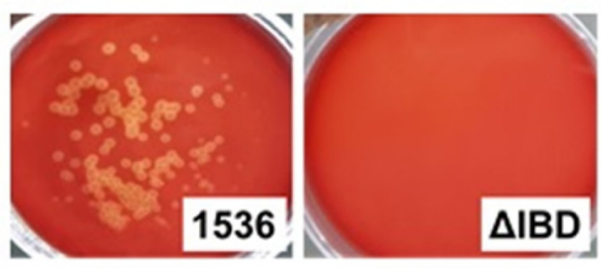

Fig. 3. Toxin secretion and hemolysis. (A) $\triangle I B D$ released lower amounts of ApxII and ApxIII toxins than 1536 in PPLO-based culture media. Western blot analysis was performed using antiApx132 antiserum for proteins precipitated from the culture media. (B) The intensities of protein bands in the western blot analysis were quantitated using the ImageJ Gel Analysis program (http://rsbweb.nih.gov/ij/). (C) In contrast to 1536, $\Delta$ IBD formed no colonies and showed no hemolytic activity on the defibrinated sheep blood agar.

the culture media was performed as described previously [9]. Based upon a western blot analysis using an anti-Apx132 antiserum, the amounts of ApxII and ApxIII proteins released into the culture media by $\triangle I B D$ were much lower than those by 1536 (Fig. 3A and 3B). These findings suggest that the inactivation of the apxIB and apxID genes inhibits the secretion of both ApxII and ApxIII.

ApxII is weakly hemolytic, but ApxIII is nonhemolytic [1]. To examine the hemolytic activity of $\triangle I B D$, it was spread on the defibrinated sheep blood agar with $250 \mu \mathrm{g} / \mathrm{mL} \beta-\mathrm{NAD}$ and incubated for $22 \mathrm{~h}$ at $37^{\circ} \mathrm{C}$. The 1536 strain showed hemolytic activity, as demonstrated by the clear zones surrounding the colonies on the defibrinated sheep blood agar (Fig. 3C). No visible colonies were observed on the $\triangle I B D$ agar. It is possible that the growth of the $\triangle \mathrm{IBD}$ colonies was delayed compared to that of the 1536 colonies and also that the $\triangle \mathrm{IBD}$ colonies were not viable on the agar.

In summary, we developed a mutant strain of App serotype 2, lacking functional $a p x I B$ and $a p x I D$ genes. The mutant strain exhibited a compromised capacity for the release of ApxII and ApxIII toxins, suggesting that the ApxIB/ApxID exporter is involved in the release of both of the bacterial toxins. Further studies are warranted to assess 
the relative specificities of the apxIB/apxID transporter to the two toxins.

\section{Acknowledgements}

This work was supported by the Seoul National University Research Grant in 2018.

\section{References}

1. Frey J. Detection, identification, and subtyping of Actinobacillus pleuropneumoniae. Methods Mol Biol 2003;216:87-95.

2. Frey J. Virulence in Actinobacillus pleuropneumoniae and RTX toxins. Trends Microbiol 1995;3:257-261.

3. Sassu EL, Bossé JT, Tobias TJ, Gottschalk M, Langford PR, Hennig-Pauka I. Update on Actinobacillus pleuropneumoniaeknowledge, gaps and challenges. Transbound Emerg Dis 2018;65 Suppl 1:72-90.

4. Bossé JT, Li Y, Sárközi R, Fodor L, Lacouture S, Gottschalk M, Casas Amoribieta M, Angen $\varnothing$, Nedbalcova K, Holden MT, Maskell DJ, Tucker AW, Wren BW, Rycroft AN, Langford PR; BRaDP1T consortium. Proposal of serovars 17 and 18 of Actinobacillus pleuropneumoniae based on serological and genotypic analysis. Vet Microbiol 2018;217:1-6.

5. Chiers K, De Waele T, Pasmans F, Ducatelle R, Haesebrouck F. Virulence factors of Actinobacillus pleuropneumoniae involved in colonization, persistence and induction of lesions in its porcine host. Vet Res 2010;41:65.

6. Prideaux CT, Lenghaus C, Krywult J, Hodgson AL. Vaccination and protection of pigs against pleuropneumonia with a vaccine strain of Actinobacillus pleuropneumoniae produced by site-specific mutagenesis of the ApxII operon. Infect Immun 1999;67:1962-1966.

7. Jansen R, Briaire J, Kamp EM, Gielkens AL, Smits MA. Structural analysis of the Actinobacillus pleuropneumoniaeRTX-toxin I (ApxI) operon. Infect Immun 1993;61:36883695

8. Park C, Ha Y, Kim S, Chae C, Ryu DY. Construction and characterization of an Actinobacillus pleuropneumoniae serotype 2 mutant lacking the Apx toxin secretion protein genes apxIIIB and apxIIID. J Vet Med Sci 2009;71:1317-1323.

9. Lee SH, Lee S, Chae C, Ryu DY. A recombinant chimera comprising the $\mathrm{R} 1$ and $\mathrm{R} 2$ repeat regions of $M$. hyopneumoniae P97 and the N-terminal region of $A$. pleuropneumoniae ApxIII elicits immune responses. BMC Vet Res 2014;10:43. 